\title{
Optimal Location of Facilities on a Network in Which Each Facility is Operating as an M/G/1 Queue
}

\section{Tomoki Hamaguchi, Koichi Nakade}

Department of Architecture, Civil Engineering and Industrial Engineering, Nagoya Institute of Technology, Japan. Email: 21418549@stn.nitech.ac.jp,nakade@nitech.ac.jp

Received June $21^{\text {st }}, 2010$; revised July $25^{\text {th }}, 2010$; accepted August $27^{\text {th }}, 2010$.

\begin{abstract}
In this paper, we consider a facility location problem in which customers and facilities are located on a network, and each facility is assumed to be operating as an M/G/1 queuing system. In many situations, the customer chooses the nearest facility to receive service. Customer satisfaction is evaluated by the probability of waiting less than or equal to a certain time for a customer that is chosen randomly from all customers who arrives to the system. By using a computational method for obtaining the probability on the waiting time, we propose the computational heuristic methods for finding the optimal location. Numerical results show the following. First, it is shown that the tabu search with an initial solution generated by random numbers gives the near-optimal solution with the highest probability among several algorithms. Second, the computation time and solution quality are not sensitive to the sharp of the service time distribution. Third, the computation time and solution quality are highly sensitive to the system utilization. Fourth, the complete enumeration might be the best solution methodology for highly utilized systems.
\end{abstract}

Keywords: Facility Location, M/G/1 Queues, Waiting Time, Tabu Search

\section{Introduction}

Facility location problems are those which decide location of facilities that meet some decision rules, given the set of demand and potential facility sites. Most of researches have developed problems for the deterministic customer demand and the fixed utilization rate of facilities. In a real situation, however, customer demand fluctuates in time, and as a result, congestion often arises at the facilities. From a customer's point of view, congestion at the facility is an undesirable situation. In addition customers usually use the nearest facility among facilities. Thus, in addition to congestion, deciding facility location by considering the distance from customer's sites to the facility leads to the improvement of customer satisfaction.

The model presented in this paper is related to research on facility location which considers stochastic demand and congestion of facility on a network. Boffey et al. [1] have reviewed various facility location models with immobile servers, where it is assumed that a finite set of customer demand and potential facility sites are given. One of stochastic facility location models on a network has been introduced in Wang et al. [2]. Each facility operates as a simple $\mathrm{M} / \mathrm{M} / 1$ queuing system in this model. Customers are assumed to travel to the nearest facility. The objective is to determine facility location minimizing the expectation of customer's total traveling time and sojourn time. Castillo et al. [3] considers two capacity choice scenarios for the optimal location of facilities with fixed servers, stochastic demand, and congestion. Each facility operates as an $\mathrm{M} / \mathrm{M} / \mathrm{s}$ queuing system. Berman et al. [4] analyzes the problem of locating a set of service facilities on a network when considering demand lost due to insufficient coverage and demand lost due to congestion. The objective is to find the facility location minimizing the number of facilities, so that the amount of demand lost from each source does not exceed a certain level. In these models, because of the complexity of the resulting model, they focus on approximate and/or heuristic solution methods. Elhedhli [5] proposes a linearization based on a simple transformation and piecewise linear approximations and an exact solution method based on cutting planes. 
One of stochastic facility models with continuously distributed demand has been introduced by Baron et al. [6]. This model has a general distribution of the demand and general arrival and service processes. Facilities are assumed to be located at an arbitrary on a plane or space. Customers are assumed to travel to the nearest open facility. Moreover, the service level constraint is imposed to ensure adequate service at the facilities. The objective is to determine the number, location, and capacity of the facilities minimizing the sum of cost on locating facilities and servers. However, in a real situation, arbitrary possible facility sites are limited and we need to select several facility sites from several potential facility sites.

In this paper, facilities and customers are located in a network assuming that it is possible only to locate them in a limited space. Each facility assumes operating as a simple $\mathrm{M} / \mathrm{G} / 1$ queuing system. In many situations, the customer chooses which facility to receive service by considering travel time to a facility. In our model, customers are assumed to travel to the nearest facility. Customer satisfaction is measured by whether or not customer's waiting time before receiving service is less than the permitted period. Thus, the objective is to find an optimal facility location maximizing the probability of waiting less than or equal to a certain time for arriving customers.

Wang et al. [2] have found an optimal approach facility location a greedy-dropping heuristic approach and tabu search approach with an initial solution generated by a greedy-dropping heuristic approach. In this paper, in addition to these approaches, we adopt a tabu search approach with an initial solution generated by random numbers. Although the probability of the objective can be represented by using Laplace transform, it is difficult to explicitly derive the probability by using Laplace inversion since the Laplace transform is very complicated. When the permitted time is given, however, it is able to compute the probability that is more than the permitted time [7]. We propose the computational heuristic methods for finding the near-optimal location by using the method described in [7].

To evaluate the proposed algorithms, we made numerical experiments. The results show that: 1) An optimal solution can be searched with the highest percentage by using a tabu search approach with an initial by random numbers, 2) that computation time and solution quality are not sensitive to the sharp of the service time distribution, 3) that computation time and solution quality are highly sensitive to the system utilization, and 4) that complete enumeration might be the best solution methodology for highly utilized systems.

The paper is organized as follows. Our model and assumption are formally introduced in Section 2. Heuristic algorithms for finding a near-optimal location are proposed in Section 3. Computational tests of these heuristics are given in Section 4. Section 5 contains concluding remarks as well as future research.

\section{Model}

\subsection{Assumptions and the Objective}

Let $N=(V, E)$ be an undirected network with a finite set of nodes $V$ and a set of $\operatorname{arcs} E$. Let $X(\subseteq V)$ be a set of customer nodes and $F(\subseteq V)$ be a set of potential facility nodes. The sets $X$ and $F$ may overlap. Let $d_{i j}$ be the shortest distance along the network between a customer node $i(\in X)$ and a potential facility node $j(\in F)$. Figure 1 shows an example of a network. We define $u=|X|$ and $v=|F|$.

Demands which are generated by customers residing at customer nodes are called service requests. Service requests at customer node $i \in X$ are assumed to occur according to a Poisson process with mean rate $\lambda_{i}$. In order to receive service, customers travel to the nearest facility from customer nodes to facility nodes along the network. If there are multiple facilities that are equal to travel time from customer nodes, customers receive service in the facility which has the lowest index among them.

We assume that each open facility hosts a single server. Services are provided on a first-in-first-out basis. Service times are supposed to be independent among customers and have according to a general distribution $G_{j}(t)$ with expectation $1 / \mu_{j}$. Thus, facilities operate as an $\mathrm{M} / \mathrm{G} / 1$ queue. The objective is to select $p$ facility nodes in $F$ to maximize the probability of waiting less than or equal to $\tau$ for a customer that is chosen randomly from all customers who arrive to the system, where $p$ is a positive integer and $\tau$ is a positive real value.

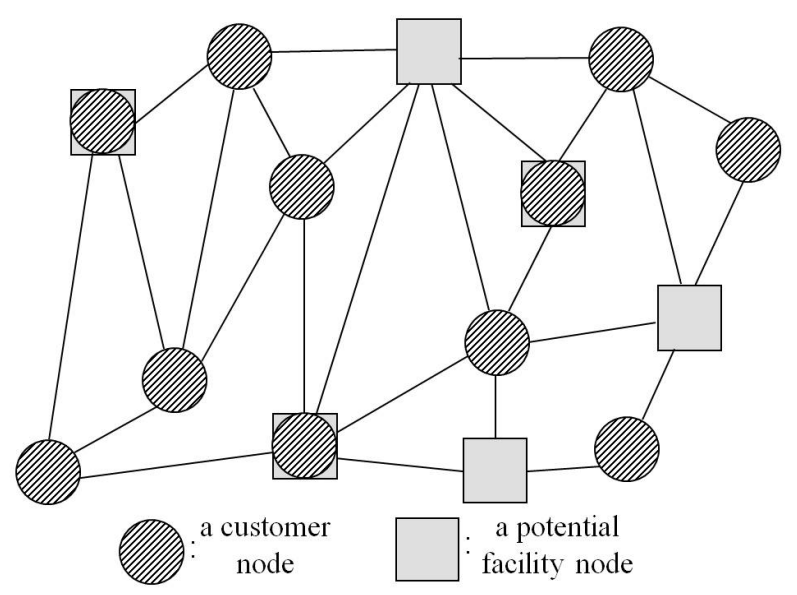

Figure 1. An example of a network. 


\subsection{Model Description}

We define the following decision variables:

$$
\begin{aligned}
& x_{i j}=\left\{\begin{array}{r}
1: \text { if a customer at node } i \in X \\
\text { uses a facility at node } j \in F, \\
0: \text { otherwise. }
\end{array}\right. \\
& y_{j}= \begin{cases}1: \text { if a facility is located at } j \in F, \\
0: \text { otherwise. }\end{cases}
\end{aligned}
$$

The objective of our model is to find an optimal facility location maximizing the probability that waiting time of customers is less than or equal to a certain time.

We define the following decision vectors $\mathbf{x}$ and $\mathbf{y}$ :

$$
\mathbf{x}=\left(\begin{array}{cccc}
x_{11} & x_{12} & \cdots & x_{1 v} \\
x_{21} & x_{22} & \cdots & x_{2 v} \\
\vdots & \vdots & \ddots & \vdots \\
& & & \\
x_{u 1} & x_{u 2} & \cdots & x_{u v}
\end{array}\right),
$$

and $\mathbf{y}=\left(y_{1}, y_{2}, \ldots, y_{v}\right)^{T}$.

Given $\mathbf{x}$ and $\mathbf{y}$, the probability in steady state that customer's waiting time $W^{j}$ at a facility $j$ is less than or equal to $t$ can be represented by $P\left\{W^{j} \leq t \mid \mathbf{x}, \mathbf{y}\right\}$. That is, the objective is given by

$$
\sum_{i \in X} \lambda_{i} \sum_{j \in F(\mathbf{y})} x_{i j} P\left\{W^{j} \leq \tau \mid \mathbf{x}, \mathbf{y}\right\},
$$

where $F(\mathbf{y})=\left\{j \in F ; y_{j}=1\right\}$.

Now we have the following mathematical programming formulation:

$$
\begin{gathered}
\operatorname{maximize} \sum_{i \in X} \lambda_{i} \sum_{j \in F(\mathbf{y})} x_{i j} P\left\{W^{j} \leq \tau \mid \mathbf{x}, \mathbf{y}\right\}, \\
\text { subject to } \sum_{j \in F} x_{i j}=1, \quad \forall i \in X, \\
\sum_{j \in F} y_{j}=p, \\
\sum_{i \in X} \lambda_{i} x_{i j}<\mu_{j} y_{j}, \quad \forall j \in F \\
\sum_{k \in F} d_{i k} x_{i k} \leq d_{i j} y_{j}+M\left(1-y_{j}\right), \\
\forall i \in X, \forall j \in F, \\
x_{i j} \in\{0,1\}, \forall i \in X, \forall j \in F, \\
y_{j} \in\{0,1\}, \forall j \in F .
\end{gathered}
$$

We now formulate constraints in our model. Constraints
(2) ensure customers certainly use a particular facility. A constraint (3) ensures facilities are located at $p$ potential facility nodes in $F$. Constraints (4) show that all customers have to receive service. Constraints in set (5) indicate that each customer receives service at the facility which has the shortest travel time from him.

Let us discuss how to compute the probability of the objective function (1). Given $y_{j}, x_{i j}$ is determined by the nearest assignment constraint described above. Consequently, the rate of service requests $\lambda_{j}(\mathbf{x})$ served at facility $j$ can be represented by $\lambda_{j}(\mathbf{x})=\sum_{i \in X} \lambda_{i} x_{i j}$. We denote the rate of utilization at the facility $j$ by $\rho_{j}(\mathbf{x})=\lambda_{j}(\mathbf{x}) / \mu_{j}$. The capacity constraint becomes $\rho_{j}(\mathbf{x})<1$ when $y_{j}=1$. We denote the complementary distribution of waiting time distribution function $W^{j}(t)=$ $W^{j}(t)=P\left(W^{j} \leq t\right)$ in steady state of an $\mathrm{M} / \mathrm{G} / 1$ system in queuing theory by $\overline{W^{j}}(t)=1-W^{j}(t)=P\left(W^{j}>t\right)$. Laplace transform ${\overline{W^{j}}}^{*}(s)$ of $\overline{W^{j}}(t)$ is given by:

$$
\begin{aligned}
& {\overline{W^{j}}}^{*}(s)=\int_{0}^{\infty} e^{-s t} P\left(W^{j}>t\right) d t \\
& =\frac{\rho_{j}(\mathbf{x}) s-\lambda_{j}(\mathbf{x})+\lambda_{j}(\mathbf{x}) G_{j}^{*}(s)}{s\left(s-\lambda_{j}(\mathbf{x})+\lambda_{j}(\mathbf{x}) G_{j}^{*}(s)\right)} .
\end{aligned}
$$

where $G_{j}^{*}(s)$ is Laplace Stieltjes transform of service time distribution $G_{j}(t)$.

Since $\bar{W}^{j}(s)$ is very complicated, it is difficult to explicitly derive $W^{j}(t)$ by using Laplace inversion. When a value $t=\tau$ is given, however, it is able to compute the probability that waiting time is more than $\tau$ [7]. In the numerical experiment of this paper, the method described in [7] is applied for computation.

\section{Heuristic Approach}

In this section, we explain heuristic approaches to compute an optimal facility location. Wang et al. [2] have found an optimal facility location using a greedy-dropping heuristic approach and tabu search approach with an initial solution generated by a greedy-dropping heuristic approach. In this paper, in addition to these two approaches, we adopt a tabu search approach with an initial solution by random numbers.

\subsection{Greedy-Dropping Heuristic Approach}

We explain the greedy-dropping heuristic approach. As preparation before applying this approach, we compute the shortest distance from all customer nodes in $X$ to all facility nodes in $F$ using Warshall-Floyd algorithm. Then, they are arranged in ascending order for each customer, that is, the facility which has the shortest distance from the customer has the higher rank for him. 
Under the assumption of our model, if the travel times from the customer to several facilities are equal, the higher rank is assumed to be assigned to a facility which has the smallest index.

After that, we compute a facility location using the greedy-dropping heuristic approach. Initially, all facilities are assumed to be located at all potential facility nodes in $F$. After determining the facility location, customers receive service at a facility which is determined by the assignment constraint (5), that is, customers receive service at the facility which has the highest rank for him among located facilities. Next, we remove facilities one by one until the number of facilities reaches $p$. If a facility is removed, the customer who selected this facility will choose the facility with the next rank for him, and as a result the numbers of customers who are served at the rest of facilities increase. Consequently, the probability that waiting time $W^{j}$ is less than or equal to $\tau$ decreases, and the value of objective function also decreases. Therefore, if there are multiple facilities for each of which the rest of facilities meets the capacity constraint (4) when it is removed, then we delete the facility which has the smallest diminution of the objective function value. The capacity constraint (4) is met if the sum of demands which are assigned to the facility does not exceed the service rate of the facility. This algorithm is repeated until one of the following stopping conditions is satisfied:

1) The number of facilities is equal to $p$.

2) The number of facilities is greater than $p$, and the capacity constraint (4) will not be satisfied even if any facility may be removed.

In case 1 , the algorithm stops at a solution which meets all constraints, whereas in case 2 the algorithm stops at the current solution. That is, the solution in case 2 also cannot meet the number of facilities constraint (3). The greedy-dropping heuristic algorithm can be described as follows.

\subsection{The Greedy-Dropping Heuristic Algorithm}

We assume that the number of customer nodes is $|X|=u$ and customer nodes are numbered from 1 to $u$, and the number of potential facility nodes is $|F|=v$ and potential facility nodes are numbered from 1 to $v$ sequentially. As a solution of facility location, let $Q(\subseteq F)$ be the set of nodes where facilities are located, and let $\psi(Q)$ be the corresponding value of objective function. Let $\varphi_{j}=\psi(Q \backslash\{j\})$ be the objective function value when facility $j$ is removed from $Q$. When facility $j$ is removed, if the capacity constraint (4) cannot be met, then set $\varphi_{j}=\psi(Q \backslash\{j\})=0$.

Preparation: First, we compute all $d_{i j}(i \in X, j \in F)$ using Warshall-Floyd algorithm. Next, because a customer at each node travels to facility which has the shortest travel time due to the closest assignment constraint, we arrange $d_{i j}$ to each customer $i$ in ascending order, facilities are ranked for each node in this order. If the distances from a customer node to facilities $j_{1}$ and $j_{2}\left(j_{1}<j_{2}\right)$ are equal, the rank shows preference for facility $j_{1}$.

Step 1: Locate facilities at all potential facility nodes, i.e., set $y_{j}=1$ for all $j \in F$ and $Q=\{1, \ldots, v\}$.

Step 2: Compute $\varphi_{j}(\forall j \in Q)$, then find $j_{0}=\operatorname{argmax}\left\{\varphi_{j}\right\}$.

If $|Q|=p+1$ and $\varphi_{j_{0}}>0$, this algorithm stops after outputting $Q \backslash\left\{j_{0}\right\}^{0}$ because the number of facilities in $Q \backslash\left\{j_{0}\right\}$ reaches $p$.

If $|Q| \geq p+1$ and $\varphi_{j}=0$ for all $j \in Q$, this algorithm stops after outputting $Q$ since the solution cannot meet the capacity constraint (4) when any facility is removed. In this case, $Q$ cannot meet the number of facilities constraint (3).

Otherwise, set $Q \leftarrow Q \backslash\left\{j_{0}\right\}$ and go to Step 2 .

\subsection{Tabu Search Approach}

In the greedy-dropping heuristic approach, it stops either at a solution that achieves a goal by reaching $|Q|=p$ facilities or that does not achieve a goal by stopping a location with $|Q|>p$ facilities. Thus, in order either to have the opportunity to investigate a high-quality solution, or to verify whether or not there is a solution in which the number of facilities is $|Q|=p$, we apply the tabu search approach.

To apply tabu search, we need an initial solution. We use a solution obtained by the greedy-dropping heuristic approach. In addition to a solution generated by an algorithm, we use a solution generated by random numbers as an initial solution. Here, a solution generated by random numbers is a one obtained by selecting $p$ facilities with equal probability using random numbers.

When using the greedy-dropping heuristic approach to derive an initial solution, we use the solution as an initial solution, even if the solution in which $|Q|>p$ is output.

After an initial solution is found, tabu search approach is implemented. We define the neighborhood $H(Q)$ of a solution $Q$ as a set of all possible pairwise facility swaps each of which is the swapping of one facility in the solution with the other facility not in the solution. For example, when $Q=\{a, b\}$ and $\bar{Q}=F \backslash Q=\{c, d\}$, the neighborhood is $H(Q)=\{(a, c),(a, d),(b, c),(b, d)\}$. At each iteration, we select a facility swap that provides the best objective function value (1). This swapping is repeated until one of the following stopping conditions is met:

1) The objective function value (1) shows no improve- 
ment at successive $K$ times.

2) Even if we carry out every facility swaps in the neighborhood, the solution cannot meet the capacity constraint (4).

In case that we use a solution of $|Q|>p$ as an initial solution, we apply the greedy-dropping heuristic approach to know whether the number of facilities can become $p$ every time the facility swap is carried out. In case that a solution $Q$ meets the above stopping condition and the number of facilities cannot be decreased, we output $Q$.

In case that the current solution $Q$ is a local optimum, it is highly likely that the solution returns to $Q$ again by continuing swaps after moving from $Q$ to the other solution $Q^{\prime}$. To avoid this, we prepare for the set of pairs of facilities called a tabu list. That is, we forbid swapping of pairs of facilities involved in this list.

To present the algorithm, we introduce the following additional notation:

- $Q^{*}$ : the best solution found so far;

- $L$ : the length of the tabu list;

- $K$ : the maximum number of successive non-improvement swaps permitted;

- $T L(i, j)$ : the number of iterations which are remained until facility pair $(i, j)$ is permitted to come up for candidates of exchange.

Note that if $T L(i, j)>0$, pair $(i, j)$ is in the tabu list and cannot be used in the next $T L(i, j)$ iterations. Using the defined notations, we denote the neighborhood of $Q$, i.e., the set of feasible facility swaps, by $H(Q)=\{(i, j): i \in Q, j \in F \backslash Q, T L(i, j)=0\}$. The tabu search algorithm is as follows.

\subsection{The Tabu Search Algorithm}

Step 1: Let $Q$ be the solution generated by the greedydropping heuristic approach or the random number. Set $Q^{*}=Q, k=0, T L(i, j)=0(\forall i, j \in F)$, and set $H(Q)=\{(i, j): i \in Q, j \in N \backslash Q, T L(i, j)=0\}$.

Step 2: If $H(Q)$ is empty, stop and output $Q^{*}$. Otherwise, swaps are tried on all pairs in $H(Q)$, then we search the facility swap $(s, t)$ in $H(Q)$ that meets the capacity constraint (4) and provides the best value of the objective function. If any facility swap in $H(Q)$ does not meet the capacity constraint (4), this algorithm stops after outputting $Q^{*}$. In this case, if $\left|Q^{*}\right|>p, Q^{*}$ cannot meet the number of facilities constraint (3).

Step 3: If $\psi(Q \cup\{t\} \backslash\{s\})>\psi(Q)$, then set $k \leftarrow 0, Q^{*} \leftarrow Q \cup\{t\} \backslash\{s\}$; otherwise, set $k \leftarrow k+1$. If $k=K$, then we have performed $K$ successive swaps that do not improve the objective function value, stop and output $Q^{*} ;$ otherwise, set $Q \leftarrow Q \cup\{t\} \backslash\{s\}$, $T L(s, t)=T L(t, s)=L \quad, \quad T L(i, j)=\max \{T L(i, j)-1,0\}$ $(\forall(i, j) \neq(s, t)$ or $(t, s))$, and $H(Q)$ is updated.
If $|Q|=p$, go to Step 2, and if $|Q|>p$, go to Step 4 .

Step 4: To check whether or not the number of facilities can decrease from $|Q|$ to $p$, we apply the greedy-dropping heuristic approach. When we apply the algorithm, as an initial location of Step 1 of this algorithm, we use the current solution $Q$. After applying the algorithm, set the derived solution as $Q$, and then go to Step 2.

Unlike the greedy-dropping heuristic approach, the approach with an initial solution obtained by the random number will generate the different solution every time. The solution obtained after the tabu search will strongly depend on the initial solution. In the numerical experiments of the next section, when we apply a tabu search with these approaches, we repeat the tabu search with different initial solutions five times, and output the solution which has the best objective function among solutions obtained after every tabu search. We can expect that the solution obtained will be improved, since tabu search approach is applied using a variety of initial solutions.

\section{Computational Results}

In this section, we numerically test heuristic approaches described in the previous section by applying various data, and discuss the results.

\subsection{Experimental Setup}

In this paper, the following three experiments are conducted by altering parameters.

1) the numbers of nodes

2) utilization rates and distributions

3) the number of potential facility nodes

Before starting experiments, we automatically generate networks by using the following method. Let $N$ be the number of total nodes in a network, and let $A$ be the number of total arcs in a network. Initially, we locate $N$ nodes by using a uniform random number on a plane region of $0 \leq x \leq X, 0 \leq y \leq Y$. Next, we select two nodes from located nodes in a random manner, and connect them by an arc. At this time, the distance between two nodes is Euclidean, and we select $A$ arcs with avoiding duplicates. Finally, by Warshall-Floyd algorithm, we check whether or not all nodes are connected. If some two nodes are not connected, we reattempt from a generation of nodes; otherwise, we conduct a numerical experiment by using the generated network. Figure 2 shows an example of a network generated automatically as $X=100, Y=100, N=200$, $A=400$ by Mathematica 7.0.

In addition, in this paper, all programs are programmed by using $\mathrm{C}$ language and compiled by using Fujitsu $\mathrm{C}$ 


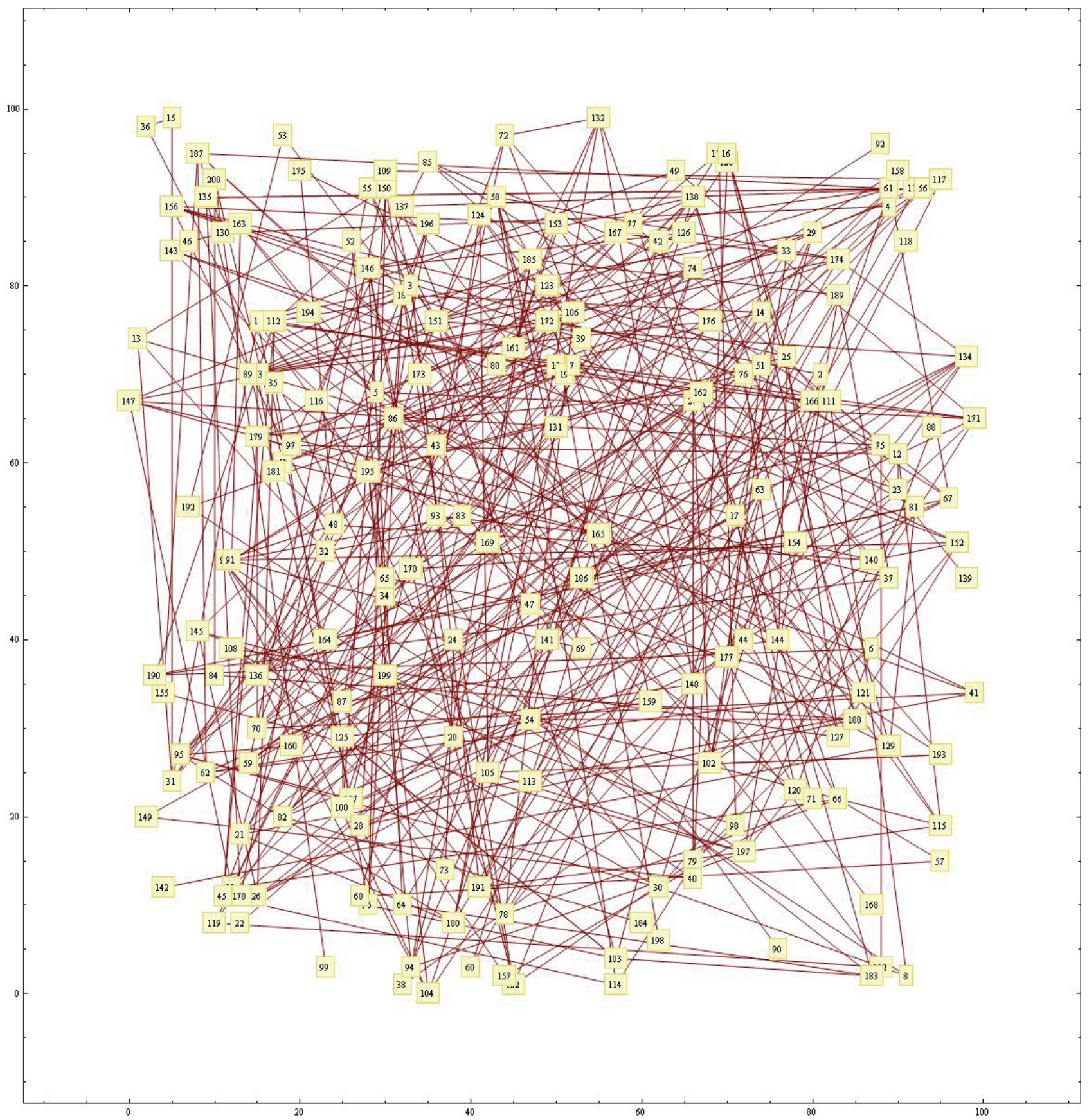

Figure 2. An example of a network using a numerical experiment.

Compiler, and executed on a $\mathrm{PC}$ with Intel(R) Core (TM)2 CPU4300 1.80GHz 3GB RAM.

First, we explain approaches and parameters used in numerical experiments. Approaches in numerical experiments are as follows:

- Exhaustive search [Combination] (COMB);

- Greedy-dropping heuristic approach (GD);

- Tabu search approach with an initial solution generated by using a greedy-dropping heuristic approach (GD-T);

- Tabu search approach with initial solutions generated by random numbers (RAND-T).

An exhaustive search is an approach that searches an optimal solution by computing objective values on possible all combinations. From now on, we call each approach by the corresponding abbreviated expressions.

As discussed in the last section, the RAND-T repeat tabu search with initial solutions generated by random 
numbers five times, and then they outputs a solution that provides the best objective function value among solutions obtained by tabu search.

We explain parameters used in experiments. Based on preliminary experimentation, the common parameters are set as follows:

- Numerical Laplace Inversion algorithm: the number of points conducted in the Laplace Inversion algorithm simultaneously is 32 ;

- Tabu search algorithm: the length of a tabu list is 7;

- Tabu search algorithm: the maximum number of successive non-improvement swaps permitted is 9 .

In all experiments, we assume that each facility has the same service rates and each customer node has the same service request rates. In addition, we use the utilization rates to determine the service request rates. For example, if the number of facilities with service rate 1 is 3 , the number of customer nodes is 10 , and the utilization rate is 0.6 , the service request rate of each customer node is set as $((1 \times 3) \times 0.6) / 10=0.18$.

In experiment 1 , we analyze the effect of the numbers of nodes. Table 1 shows parameters used in experiment 1.

In experiment 2 , we analyze the influence in the case that utilization rates and distributions are altered. Table 2 shows parameters used in experiment 2 .

In experiment 3 , we analyze the influence in the case that the number of potential facility nodes is altered. Table 3 shows parameters used in experiment 3 .

As combinations of parameters described above, experiment 1 has 5 combinations. Similarly, experiment 2 has 24 combinations and experiment 3 has 4 combinations. For each of 33 total combinations of parameter values, 10 problem instances are generated, leading to a total of 330 problem instances.

\subsection{Experimental Results}

Before showing the experimental results, we explain

Table 1. Parameters used in experiment 1.

\begin{tabular}{cc} 
Total nodes & $100,200,300,400,500$ \\
Potential facility nodes & 30 \\
Customer nodes & Total nodes - 300 \\
Empty nodes & 0 \\
Facilities & 5 \\
Distribution & Erlang (degree: 2$)$ \\
Utilization rate & 0.6 \\
Service rate & 1 \\
Waiting time & 1 \\
\hline
\end{tabular}

Table 2. Parameters used in experiment 2.

\begin{tabular}{|c|c|}
\hline Total nodes & 500 \\
\hline Potential facility nodes & 30 \\
\hline Customer nodes & 470 \\
\hline Empty nodes & 0 \\
\hline Facilities & 5 \\
\hline Distribution & $\begin{array}{c}\text { Exponential } \\
\text { Erlang (degree: 2) } \\
\text { Erlang (degree: } 3 \text { ) } \\
\text { Erlang (degree: 5) }\end{array}$ \\
\hline Utilization rate & $0.6,0.9$ \\
\hline Service rate & 1 \\
\hline Waiting time & $1,3,10$ \\
\hline \multicolumn{2}{|c|}{ Table 3. Parameters used in experiment 3.} \\
\hline Potential facility nodes & $20,25,30,35$ \\
\hline Customer nodes & 500 \\
\hline Total nodes & $\begin{array}{l}\text { Customer nodes }+ \\
\text { Potential facility nodes }\end{array}$ \\
\hline Empty nodes & 0 \\
\hline Facilities & 5 \\
\hline Distribution & Erlang (degree: 2) \\
\hline Utilization rate & 0.6 \\
\hline Service rate & 1 \\
\hline Waiting time & 1 \\
\hline
\end{tabular}

following performance measures estimated by experiments.

1) Optimal rate (\%): a fraction of instances in which the obtained solutions coincide with optimal solutions obtained by the COMB.

2) Relative error (\%): an average of rates obtained by dividing absolute values of differences between the corresponding optimal objective values by the COMB and objective values by each algorithm by the former values.

3) Average computation time (seconds): average computation time until the solutions are searched by each algorithm after data of network is given.

In experiment 2, we also discuss fractions of experiments that reach $p$ facilities for the solutions because the solutions obtained by heuristics do not reach targets on the number of facilities with high probability in some cases.

\subsubsection{Experiment 1}

We consider the result in the case that the number of nodes is altered. Figures $\mathbf{3}$ and $\mathbf{4}$ show average computa- 


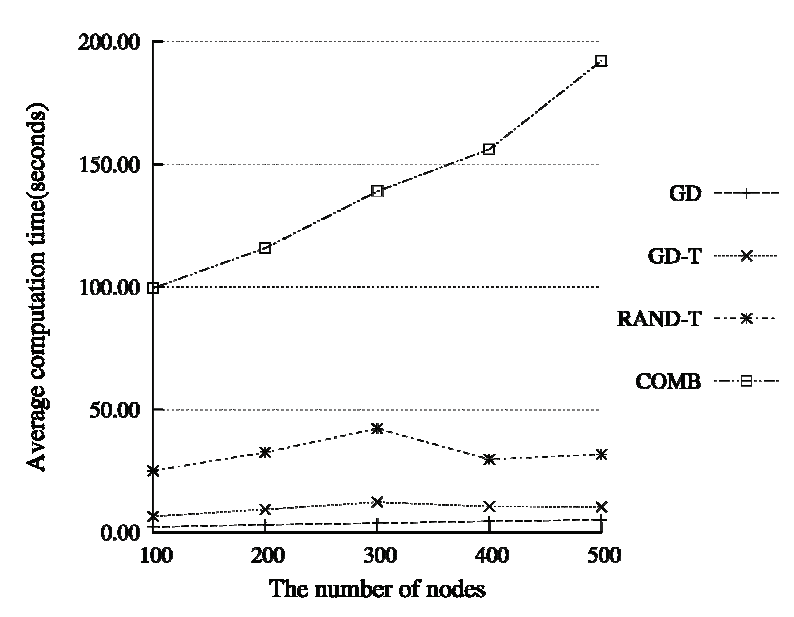

Figure 3. Average computation time in experiment 1.

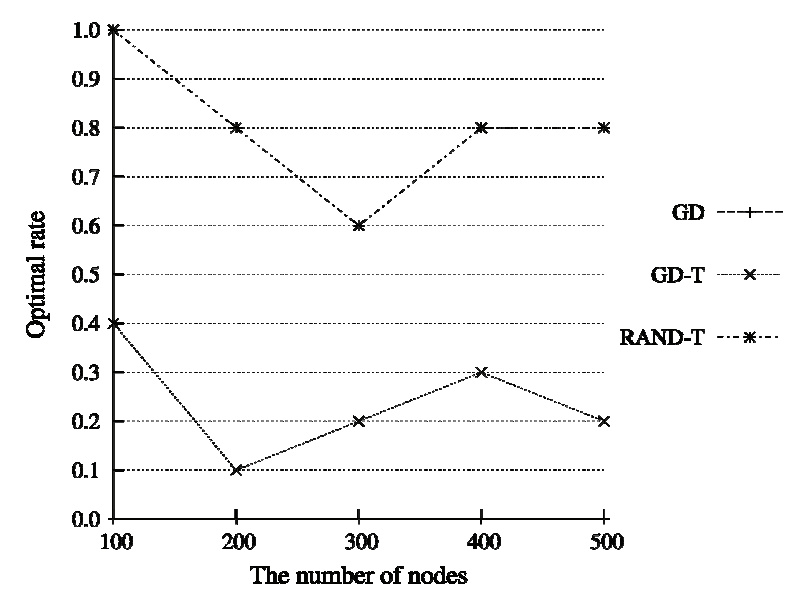

Figure 4. Optimal rates in experiment 1.

tion time and the optimal rate, respectively. Table 4 shows average values of relative errors obtained by each algorithm. Relative errors are average values among experiments with 100 to 500 nodes. Note that they are almost constant on the number of nodes, and that all solutions in this experiment have $p(=5)$ facilities.

From Figure 3, we can see that average computation time of the COMB is increasing in the number of nodes. By contrast, average computation times of the other algorithms are not much increasing. Because the number of potential facility nodes is set to 30 , the increase of the number of nodes implies the increase of the number of customer nodes. Consequently, if the number of nodes increases, the computation time necessary for determining a service facility for each customer also increases. In the case of the COMB, because the increase of the number of nodes has an influence on all combinations, the running time has increased greatly. On the other hand, because the other algorithms update solutions that meet conditions by removing and exchanging, they can search for the number of combinations that is much fewer than the number of combinations in the COMB. As a result, the increase of the number of nodes has little influence on the running time. Because the RAND-T searches solutions five times, that average time is longer than that of GD.

From Figure 4, we can see that the RAND-T typically finds the optimal solution. The fraction that it can search the optimal solution is about $80 \%$. It is because the tabu search approach is conducted to a variety of directions thanks to a diversity of initial solutions. But, the GD cannot search optimal solutions at all. The GD-T also can search solutions only the average of about $30 \%$. From Table 4, however, the relative errors of the GD is $4 \%$, and GD-T has smaller errors. This means that most of cases in the GD and GD-T missed to escape from the local optimal solution and stopped to search before reaching the optimal solution in the tabu search.

\subsubsection{Experiment 2}

We show the experimental result in the case that service time distributions and utilization rates are altered. Although we conducted the experiment by using an exponential distribution and Erlang distributions (degree: 2,3,5), there is not so much of differences between Erlang distributions. Thus we consider the cases of an exponential distribution and an Erlang distribution (degree: 3). Table 5

Table 4. Average of relative errors in experiment 1.

\begin{tabular}{cccc}
\hline & GD & GD-T & RAND-T \\
\hline Average of relative errors & 0.039 & 0.016 & 0.001 \\
\hline
\end{tabular}

Table 5. Optimal rate in the case that utilization rates and distributions are altered.

\begin{tabular}{ccccc}
\hline & Waiting time & GD & GD-T & RAND-T \\
\hline Exponential: 0.6 & 1 & 0 & 0.2 & 0.6 \\
& 3 & 0 & 0.2 & 0.7 \\
& 10 & 0 & 0.3 & 0.9 \\
Exponential: 0.9 & 1 & 0 & 0.2 & 0.2 \\
& 3 & 0 & 0.3 & 0.1 \\
& 10 & 0 & 0.2 & 0 \\
Erlang(3): 0.6 & 3 & 0 & 0.2 & 0.8 \\
& 1 & 0 & 0.2 & 0.8 \\
& 1 & 0 & 0.3 & 0.5 \\
Erlang(3): 0.9 & 3 & 0 & 0.2 & 0.1 \\
& 10 & 0 & 0.3 & 0 \\
\hline
\end{tabular}


shows the optimal rate in the case that utilization rates and distributions are altered, and Table 6 shows the average computation time.

Table 7 shows rates on searching solutions with targets on the number of facilities generated by RAND-T. In RAND-T, we conduct tabu search with different initial solutions five times for each instance, and we output a solution that provides the best objective function value of them. Thus, because we conduct experiments by using the same parameters, Table 7 shows average of 50 total experimental results. Table 8 shows rates of experiments succeeding in finding solutions with targets of the number of facilities by the other algorithms when distributions and utilizations are altered.

From Table 5, for any utilization rates and distributions, optimal rates of GD and GD-T are almost the same as in Experiment 1 . They are not influenced by $\tau$. Consequently, we can see that the distribution (Exponential or Erlang) has almost no impact on optimal rates.

Table 6. Average computation time in the case that utilization rates and distributions are altered.

\begin{tabular}{cccccc}
\hline & $\begin{array}{c}\text { Waiting } \\
\text { time }\end{array}$ & GD & GD-T & RAND-T & COMB \\
\hline Exponential: & 1 & 4.586 & 9.407 & 30.505 & 176.562 \\
0.6 & 3 & 4.595 & 9.473 & 28.199 & 176.566 \\
& 10 & 4.565 & 10.506 & 28.420 & 176.538 \\
& 1 & 4.530 & 5.964 & 0.144 & 12.264 \\
Exponential: & 3 & 4.527 & 5.866 & 0.109 & 12.253 \\
0.9 & 10 & 4.497 & 5.597 & 0.108 & 12.324 \\
& 1 & 5.412 & 10.981 & 33.192 & 206.807 \\
$\begin{array}{c}\text { Erlang(3): } \\
0.6\end{array}$ & 3 & 5.414 & 11.053 & 35.014 & 206.758 \\
& 10 & 5.381 & 12.249 & 36.438 & 206.691 \\
Erlang(3): & 1 & 5.350 & 6.686 & 0.117 & 12.633 \\
0.9 & 3 & 5.339 & 6.877 & 0.120 & 12.681 \\
& 10 & 5.315 & 6.567 & 0.153 & 12.342 \\
\hline
\end{tabular}

Table 7. Rates of experiments reaching targets of the number of facilities in solutions generated RAND-T.

\begin{tabular}{cccc}
\hline & Waiting time & $\begin{array}{c}\text { the utilization } \\
\text { rate } 0.6\end{array}$ & $\begin{array}{c}\text { the utilization } \\
\text { rate } 0.9\end{array}$ \\
\hline \multirow{3}{*}{ Exponential } & 1 & 0.94 & 0.04 \\
& 3 & 0.88 & 0.04 \\
& 10 & 0.92 & 0.06 \\
Erlang(3) & 1 & 0.96 & 0.02 \\
& 3 & 0.92 & 0.02 \\
& 10 & 0.96 & 0.06 \\
\hline
\end{tabular}

Table 8. Rates of experiments finding the solution that achieves targets of the number of facilities in the case that utilization rates and distributions are altered.

\begin{tabular}{cccc}
\hline & Waiting time & GD & GD-T \\
\hline Exponential: 0.6 & 1 & 1.0 & 1.0 \\
& 3 & 1.0 & 1.0 \\
& 10 & 1.0 & 1.0 \\
Exponential: 0.9 & 1 & 0.4 & 0.7 \\
& 3 & 0.3 & 0.7 \\
Erlang(3): 0.6 & 10 & 0.3 & 0.7 \\
& 1 & 1.0 & 1.0 \\
& 3 & 1.0 & 1.0 \\
Erlang(3): 0.9 & 10 & 1.0 & 1.0 \\
& 3 & 0.4 & 0.7 \\
& 10 & 0.3 & 0.7 \\
\hline
\end{tabular}

From Table 6, average computation time also are not influenced by $\tau$. This means that the distribution have little effect on the searching. As utilization rates are large, however, Tables 5 and $\mathbf{6}$ show that we can hardly find solutions even if we search from a variety of directions in a random manner. In the case that the utilization rate is 0.6 , we can almost find solutions that meet targets of the number of facilities, but in the case that the utilization rate is 0.9 , we were hardly able to find solutions that meet targets of the number of facilities. This is because the number of the solutions that meet the capacity constraint (4) decreases in the utilization rapidly. As shown in Table 8 the same phenomenon appears in the other algorithms. Thus, in the case that the utilization rate is too large, it is difficult for our algorithms to search optimal solutions or near-optimal solutions. In the case that the utilization rate is 0.9 , however, we can see that the COMB can search optimal solutions quickly. The computation is done quickly because the COMB skips the computation with respect to the solution that does not meet the capacity constraint (4) as a large majority of the solutions cannot meet this constraint. Thus, in the case that the utilization rate is high, the COMB is the best algorithm in this instance.

\subsubsection{Experiment 3}

We consider the results in the case that the number of potential facility nodes is altered. Targets of the number of facilities are set to 5 . Figure 5 shows optimal rates in the case that the numbers of potential facility nodes are altered, and Figure 6 and Table 9 show average computation time and relative errors respectively. 


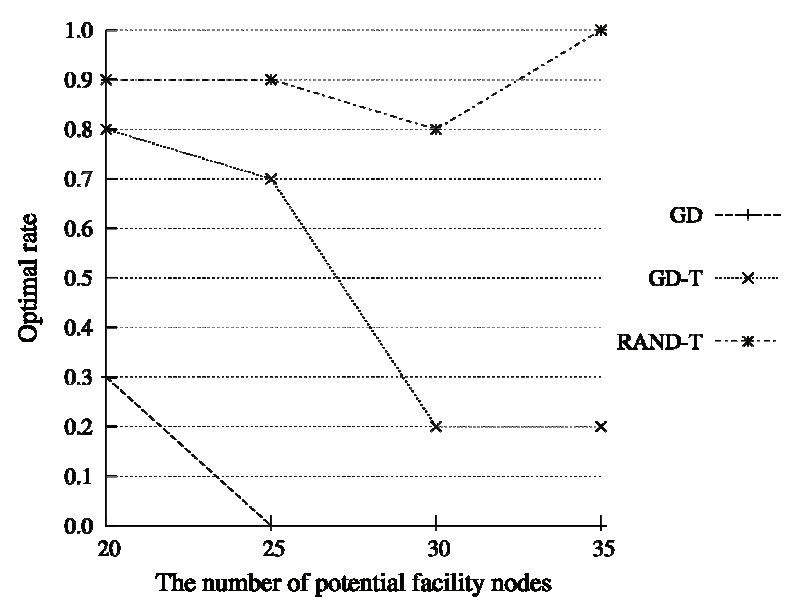

Figure 5. Optimal rates in the case that potential facility nodes are altered (A target of the number of facilities: 5 ).

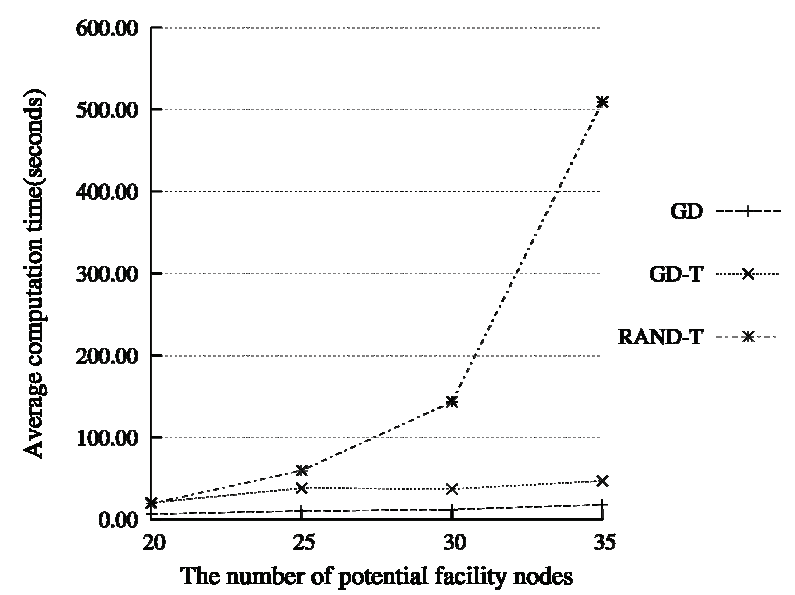

Figure 6. Average computation time in the case that potential facility nodes are altered (A target of the number of facilities: 5).

Table 9. Relative errors in the case that potential facility nodes are altered (A target of the number of facilities: 5).

\begin{tabular}{lccc}
\hline & GD & GD-T & RAND-T \\
\hline Potential facility nodes: 20 & 0.01 & 0.00 & 0.00 \\
Potential facility nodes: 25 & 0.03 & 0.01 & 0.00 \\
Potential facility nodes: 30 & 0.02 & 0.00 & 0.00 \\
Potential facility nodes: 35 & 0.02 & 0.00 & 0.00 \\
\hline
\end{tabular}

From Figure 5, we can see that the GD was hardly able to search the optimal solution. The GD-T tends to decrease optimal rates as the number of facilities increases. We can see that the optimal rates of the RAND-T are higher than those of the other algorithm. From Table 9, even if all algorithms increase the number of potential facility nodes, relative errors hardly alters and they are very small. The relative error of the RAND-T is smaller than the other algorithm although the optimal rate is greater. Thus, it can be concluded that the RAND-T is the best algorithm to search an optimal facility location in our model.

\subsection{Analysis of Path Searched at the Tabu Algorithm}

The GD-T moves nearer to the optimal solution in comparison to the solution generated by GD, but do not reach the optimal solutions in many cases. We discuss the reason why their approaches cannot search an optimal solution.

Figure 7 shows a part of the path search of the GD-T, which searches in the order of from the facility swap 1 to the facility swap 5 . The origin of each arrow shows the facility that is removed by exchange, and the end of the arrow shows the facility that opens by exchange. From Figure 7, we can see that the location returns to the first location by the successive exchange from the facility swap 1 to the facility swap 3 . That is because this algorithm searches toward the first solution by improving the solution by the facility swaps 2 and 3 after it is updated to the not-improved solution by conducting the facility swap 1. In addition, because the algorithm cannot exchange facilities by swap 1 in tabu list, the solution returns to the initial location by conducting the facility swap 5 after the solution is updated to the not-improved solution by conducting the facility swap 4 . After that, because the swap used so far cannot be conducted, this algorithm exchanges facilities by the other swaps included in the neighborhood, and then this algorithm stops by reaching the maximum number of successive nonimprovement swaps permitted. That is one of the reasons why the algorithm sometimes accepts to update toward the worse solution.

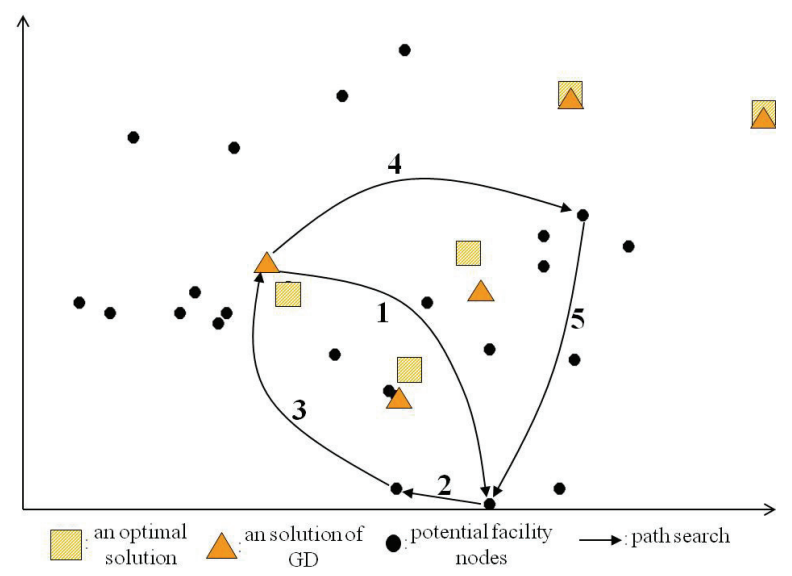

Figure 7. A part of the path search of the tabu search algorithm. 


\section{Conclusions and Future Work}

This paper focuses on a facility location problem with stochastic customer demand and congestion where customers travel to facilities to receive services. We explain a model that located customers and facilities on network and provide services at each facility operating as an $\mathrm{M} / \mathrm{G} / 1$ queuing system. The objective of our model is to search an optimal facility location maximizing the probability of waiting less than or equal to a certain time for a customer that is chosen randomly from all customers who arrive to the system. In order to search an optimal location, we propose three heuristic algorithms: GD, GD$\mathrm{T}$, and RAND-T. We conduct numerical experiments by using these algorithms and the COMB and discuss the properties of these algorithms.

Based on the results of numerical experiments, we can search an optimal solution with the highest percentage by using the RAND-T in many cases. However, in the case that utilization rates are high, we need to search an optimal solution by using the COMB because it is difficult for the other three algorithms to search an optimal solution. The tabu search approach has a property that it returns to the first location through several facilities, which results in stops at the local optimal solution. Although we assume that the number of servers at each facility is set as one, multiple servers will be considered as more realistic problems. These are also left in the future.

\section{REFERENCES}

[1] R. Boffey, R. Galvao and L. Espejo, "A Review of Congestion Models in the Location of Facilities with Immobile Servers," European Journal of Operational Research, Vol. 178, No. 3, 2007, pp. 643-662.

[2] Q. Wang, R. Batta and C. Rump, "Algorithms for a Facility Location Problem with Stochastic Customer Demand and Immobile Servers," Annals of Operations Research, Vol. 111, No. 1-4, 2002, pp. 17-34.

[3] I. Castillo, A. Ingolfsson and T. Sim, "Social Optimal Location of Facilities with Fixed Servers, Stochastic Demand, and Congestion," Production and Operations Management, Vol. 18, No. 6, 2010, pp. 721-736.

[4] O. Berman, D. Krass and J. Wang, "Locating Service Facilities to Reduce Lost Demand," IIE Transactions, Vol. 38, No. 11, 2006, pp. 33-946.

[5] E. Elhedhli, "Service System Design with Immobile Servers, Stochastic Demand, and Congestion," Manufacturing and Service Operations Management, Vol. 8, No. 1, 2006, pp. 92-97.

[6] O. Baron, O. Berman and D. Krass, "Facility Location with Stochastic Demand and Constraints on Waiting Time," Manufacturing and Service Operations Management, Vol. 10, No. 3, 2008, pp. 484-505.

[7] H. Tijms, "A First Course in Stochastic Models," Wiley, New York, 2003. 